\title{
An Analysis on the Application of P2P in Network
}

\author{
Yongmei Zhang \\ Network Center, \\ China West Normal University, \\ Nanchong, Sichuan, China. \\ E-mail: zymface@163.com
}

\begin{abstract}
Currently, P2P is a very popular network model. Compared with the traditional model, it bears the advantages of high transmission speed and no centralization etc, and it performs much better in the cyber source utilization and server bottlenecks elimination. This article, starting from the basic principle of $\mathrm{P} 2 \mathrm{P}$ technology, proceeds with the structure modes and characteristics of P2P to combine it with the application of campus network, so as to analyze the current problems in campus network and to give the solutions to such problems of P2P.
\end{abstract}

\section{Keywords-P2P;P2P structure model; campus network}

\section{THE CONCEPT OF P2P AND NETWORKS STRUCTURE}

\section{A. The concept of $\mathrm{P} 2 \mathrm{P}$}

$\mathrm{P} 2 \mathrm{P}$ is a 'point to point, end to end' distributing network, also known as peer-to-peer network. It is different from the $\mathrm{B} / \mathrm{S}$ or $\mathrm{C} / \mathrm{S}$ conventional mode used currently. Each node in the P2P network is equal, and each node can request the services and also provide the service. Each node can exchange resources and services directly without adopting the server. Thereby, the direct interconnection between nodes is realized, and P2P has been widely applied in the campus network.

\section{B. The network structure of $P 2 P$}

$\mathrm{P} 2 \mathrm{P}$ is a network system of resource distribution and utilization and sharing. It has the advantages of sharing the capacity and resources of each node. The development of P2P network has experienced three stages: the centralized $\mathrm{P} 2 \mathrm{P}$, distributed $\mathrm{P} 2 \mathrm{P}$ and hybrid $\mathrm{P} 2 \mathrm{P}$.

1) Centralized $P 2 P$ network.

The topology of centralized P2P model is similar to $\mathrm{B} / \mathrm{S}$, which requires a central server to connect. The center server can collect and integrate all the resources from every user's personal computer and edit them to form a huge complete index directory. The user can check this index from his computer to choose their favorite resource to download. The centralized P2P network structure has a central server, but it is not the same as the traditional C/S model. In the traditional $\mathrm{C} / \mathrm{S}$ mode, all the information resources obtained through searching are stored in the central server. The users must pass through the central server to read information. Each user's PC can not exchange information mutually. The centralized P2P network is different. The information resources are stored in the user's PCs, while the server only plays a role as retention of information. In this mode, a mutual exchange of information resource can occur among all users of PC and among PC users and the server. This model has the advantages of high search efficiency, easy management, with the characteristics of centralization; the drawback is the server burdens heavy loads and there may be a problem of single point failure. The network topology is shown as in figure 1 .

\section{2) Distributed $P 2 P$ network}

The Distributed P2P network has no central server, and each node each connects directly and accesses to network randomly. Each node has similar function and is equal in status. Since there is no center server to store the index information of shared resources, a node must use the request packet to search the entire network to obtain the cyber source. Therefore, many invalid packets will be produced. The disadvantage of this model is to solve the problem of single point failure. The disadvantage exists in the occupation of large bandwidth, lacking fast searching ability and strong expansibility. The network topology is shown as in Figure 2

3) Hybrid P2P network

The hybrid P2P network is born on the basis of the previous two, so it has obvious progress in the ability and speed of processing users' request. It divides users' nodes into three categories: the user node, the search node and the index node. After classification, the nodes can function according to the categories of the work contents. User nodes have no distinction from the previous two; search nodes are responsible for searching for the list of files on the whole network, to have a complete understanding of the information; index nodes are to arrange and store the information searched by the search nodes, so that the users can find a particular resource quickly. Hybrid P2P combines the advantages of centralized P2P and distributed P2P. The design and processing capacity have been further optimized, and it is currently the most popular used model. The network topology is shown as in Figure 3

\section{The characteristics of $P 2 P$ network}

\section{1) Strong expansibility}

Each node in the P2P network is equal. The user joins the same shared system through the installation of the software. 
The more users add, the larger the network scale is. Each user has own resources, so more downloading resources are available for users. Thus, it has extremely strong expansibility.

\section{2) High robustness}

The P2P network is highly resistant to attack and fault. Because the service is dispersed in all nodes, part of the destruction will not affect a wide range of network operation, so it avoids the single point failure and improve the network robustness.

\section{3) High performance}

Performance advantage is an important reason for the wide attention on P2P. The P2P framework can effectively use a large number of ordinary nodes spread in the Internet to distribute the computation or storage data to all nodes. The unused computing power or storage spaces are used to achieve high performance of computing and storage. By using idle resources of the Internet, we can enjoy a lower cost with higher computing and storage capacity. In addition, P2P network applies automatic computing technique, which greatly reduces the operator's operating costs and improves the cost-efficiency.

\section{4) Load balance}

Each node in P2P network environment is both a client and a server. It reduces the requirements on the computing power and storage capacity in traditional structure of GS server. At the same time, as resources are distributed across multiple nodes, the better load balance is realized.

\section{P2P APPLICATIONS IN CAMPUS NETWORK}

\section{A. The application of streaming media}

$\mathrm{P} 2 \mathrm{P}$ technology of streaming media is different from the traditional streaming media because the user in the playing process can not only obtain the media stream from the streaming media serve but also from other users. At the same time, the user can also provide their own media stream to others. The P2P streaming media system can be divided into direct broadcast system and VOD system in accordance with its broadcast mode. Broadcast system is relatively simple, rapid developed, but development speed is relatively slow in VOD system. Mainly because of the high interactivity requirements in VOD system, more complex and higher degree difficulties may appear. Therefore, the streaming media system in the campus network application can be a very good solution to video broadcast discontinuous problems

\section{B. File sharing}

In the traditional Web mode, it needs to upload files to a specific site to achieve the exchange of documents, and the user goes to the website to search the needed file, and download. It is not convenient. However, because under the P2P network any of the two PC machines can directly interact and share resources, the user only needs to position resources required through different query mechanism and then connect and download the files needed.

\section{Search engine}

Because P2P technology is not limited by servers, it can give full play to direct dynamic characteristics of peer-topeer connection between nodes. The information retrieval is more targeted, and the renewal cycle is shorter. P2P resource sharing technology can make full use of the largescale distribution of information, making up the shortcoming in the traditional search engines which can not mine information with a great depth. Based on P2P, the dispersion of P2P network makes information retrieval into the terminal equipment of dynamic storage of massive information, thus changing the phenomenon that the traditional search engines can only retrieve the static site status.

\section{The sharing of computing and storage capacity}

In computing, after receiving the calculation task, the P2P network node is not confined to their surplus computing power but to search every node in the same network, so as to view the other node residual computing power situation. After computing the whole network node residual statistics, based on the actual situation, the tasks are divided into many small fragments and assigned to a spare computing capacity of the other nodes. Each node finishes their assigned tasks after the back calculation, and then transmits it to the originally task nodes. This computation can fully integrate unused computing capacity and resources of every node in the network, and it greatly improves the overall computational ability. In the term of storage capacity, since the P2P mode has a lot of user nodes and each user node has a certain amount of storage capacity, the storage capacity in the mutual sharing of resources has been greatly improved. Every resource has one or more providers, so that one node's exit will not cause great impact on the overall resources or even no impact, let along the paralysis of whole network.

\section{E. Data storage}

$\mathrm{P} 2 \mathrm{P}$ distributing storage system is used for a peer-topeer network data storage system. It can provide file access function with high efficiency and load balance. In the network, to decentralize the stored object, but not placing on a specific server, will reduce the burden on the server and increase the reliability of data transmission and speed.

\section{F. Real-time communication}

Real-time communication is similar to the QQ instant messaging software. two or more users are using voice, text, document to communicate rapidly and directly, easier than the similar PC network communication equipment ( such as PDA, mobile phone), and it does not depend on the equipment can identify users. 


\section{G. Collaborative work}

Collaborative work allows multiple users to utilize collaborative computing platform to complete a task. In the traditional Web model, then the burden of the server is heavy and cost is expensive. Yet to adopt P2P technology can easily set up the user's real-time communication and information transmission in the network, to avoid the central server generating the network and processing delay and the bottleneck of performance, to realize collaborative work more efficiently and conveniently

\section{THE PROBLEMS OF P2P APPLICATION IN THE CAMPUS} NETWORK

Although P2P application has many technical advantages in campus network, the problem in the specific application can not be ignored, and these problems are just brought about by the technical advantages. They are specifically manifested in the following aspects:

\section{A. Occupying bandwidth}

Increasing P2P applications bring very great pressure to the of campus network. Some of the P2P software occupants export resources unrestrainedly, resulting in a long-term state of congestion. The school spends a lot of money in the bandwidth, but the actual use has not been effectively guaranteed. Sudden increases in Network traffic also causes certain effect on the campus network of commonly used internal system, such as office automation system, network aided teaching system, video on demand system

\section{B. Poor safety}

With the wider application of computer network, the computer virus threat to information security is increasing. The facilitating sharing and fast selection mechanism under P2P environment provide more invasion opportunities for some network virus, which will greatly threaten the safety of P2P network. Some people make use of existing P2P system vulnerabilities to attack the personal computers of campus network users, stealing confidential information, even destructing, and therefore causing incalculable loss.

\section{Garbage information}

Due to too many users and the lack of a unified management, it is very difficult to sort a list of search results. Therefore, when a user searches, he will get a great deal of information with a few useful but most other may belong to the rubbish information. The users will inevitably fall into a vast sea of junk information.

\section{Copyright}

Essentially speaking, P2P presents the users an unlimited space. All the behavior of P2P network all belong to the individual user. The users can freely exchange valuable resource as they like, so most of the P2P services will be inevitably conflict with intellectual property rights.

\section{THE MANAGEMENT STRATEGY OF P2P CAMPUS NETWORK APPLICATION}

Aiming at the problems brought up by P2P technology, such as occupying bandwidth, poor network security, rubbish information, copyright infringement and other issues, we propose the following solution strategy:

A. To control the flow, we can use deep packet inspection technology and streaming feature recognition technology through the port, protocol feature code, and we can control the flow by traffic identification billing, limiting the maximum number of connections and the TCP/UDP port filtering means.

$B$. On the security of campus network, we can use P2P server IP address filtering to prohibit some bad sites, in order to limit the malicious P2P application; we can also use the technology of artificial intelligence, expert database technology and other means to stop spam problems, so as to make it easier to identify the virus, Trojan program to reduce the malicious program propagation in networks.

C. In terms of the violation of intellectual property rights, illegal transfer of information and other issues, those non technical means, such as legislation, regulations, realname access can be adopted.

\section{CONCLUSION}

P2P technology sets off upsurge to the network nowadays. The applications based on this technology have covered various fields over the Internet, especially for a revolutionary improvement in the university campus network Meanwhile, P2P technology is like a two-edged sword. It also brings a series of new problems to the campus network. How to reduce negative impact and give full play to the role of $\mathrm{P} 2 \mathrm{P}$ in colleges and universities campus network will be a task that deserves our research in the future

\section{REFERENCES}

[1] Napster[EB/OL]. [2009-10-08]. http: //www. napster. com.

[2] Gnutella[EB/OL]. [2009-10-08]. http: //www. napster. com.

[3] P2P Routing. http://www.cs.wm.edu/ bryan/, 2007.5

[4] P2P Working Group Home Page[EB/OL].http://www.peer-topeerwg.org,2002.

[5] Chong Gang, Wang Boli. Peer to Peer Overlay Networks: A survey. CoumputerNetworks, 2003, 4(1):23 50

[6] A.Jobhston. SIP P2P and Internet Comunications. Draft-johnstionsipping-p2p-ipcom-00, 2005:23 47

[7] D.Bryan, C.Jennings. A P2P Approach to SIP Registration. draftbryan-sipping-p2p-00, 2005: 33 62.

[8] The Jxta Solution to P2P Suns New Network Computing Plat-form Establishes a Base Infrastructure for Peer-to-Peer App-lication Development. http://ww.javaworld.com/javaworld/jw -10-2001/jw1019-jxta_p.html

[9] Peer to Peer Application Development:Cracking the Code byHungry Minds. Deamtech, http://www.a-mazon. com/exec/obidos/tg/de-tail/.

[10] Stoica, R.Morris, D.Karger, et al. Chord: A scalable peer-topeer lookup service forInternet applications. IEEE Internet Computing, 2001, 17(3):35 59 


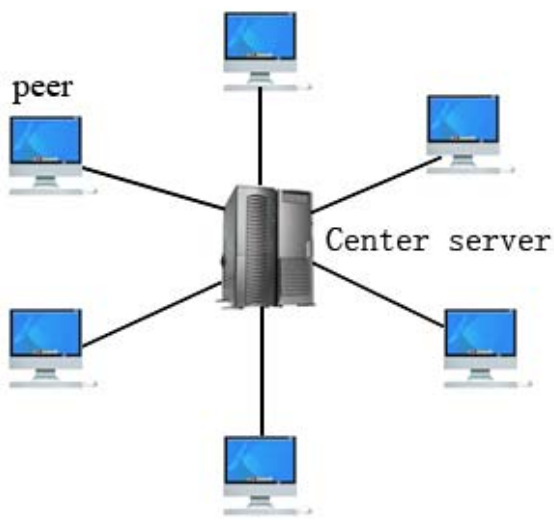

Figure 1. Centralized P2P network

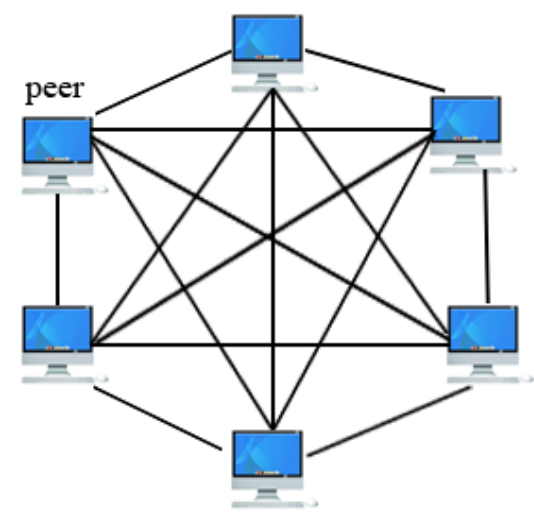

Figure 2. Distributed P2P network

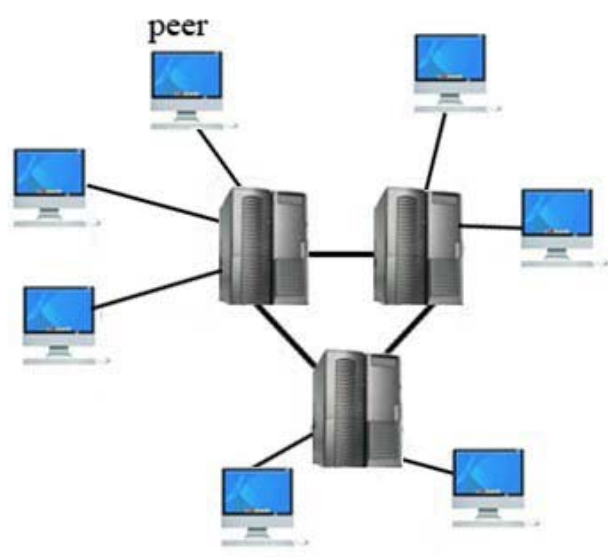

Figure 3. Hybrid P2P network 